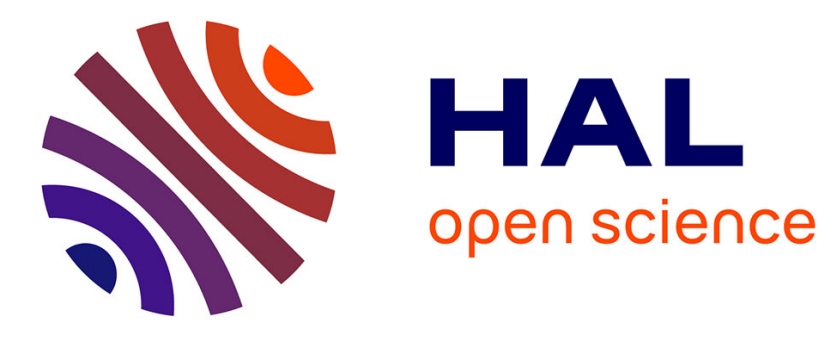

\title{
When Analog Meets Digital: Source-Encoded Physical-Layer Network Coding
}

Raphaël Naves, Gentian Jakllari, Hicham Khalife, Vania Conan, André-Luc Beylot

\section{- To cite this version:}

Raphaël Naves, Gentian Jakllari, Hicham Khalife, Vania Conan, André-Luc Beylot. When Analog Meets Digital: Source-Encoded Physical-Layer Network Coding. IEEE 19th International Symposium on "A World of Wireless, Mobile and Multimedia Networks" (WoWMoM 2018), Jun 2018, Chania, Greece. pp.1-9, 10.1109/WoWMoM.2018.8449810 . hal-03023412

\section{HAL Id: hal-03023412 \\ https://hal.science/hal-03023412}

Submitted on 1 Dec 2020

HAL is a multi-disciplinary open access archive for the deposit and dissemination of scientific research documents, whether they are published or not. The documents may come from teaching and research institutions in France or abroad, or from public or private research centers.
L'archive ouverte pluridisciplinaire HAL, est destinée au dépôt et à la diffusion de documents scientifiques de niveau recherche, publiés ou non, émanant des établissements d'enseignement et de recherche français ou étrangers, des laboratoires publics ou privés. 


\title{
When Analog Meets Digital: Source-Encoded Physical-Layer Network Coding
}

\author{
Raphaël Naves $^{\dagger *}$, Gentian Jakllari*, Hicham Khalifé ${ }^{\dagger}$, Vania Conan ${ }^{\dagger}$ and André-Luc Beylot \\ ${ }^{\dagger}$ Thales Communications \& Security - France, Email: name.surname@thalesgroup.com \\ *IRIT-INPT/ENSEEIHT - France, Email:name.surname@enseeiht.fr
}

\begin{abstract}
We revisit Physical-Layer Network Coding (PLNC) and the reasons preventing it from becoming a staple in wireless networks. We identify its strong coupling to the Two-Way Relay Channel (TWRC) as key among them due to its requiring crossing traffic flows and two-hop node coordination. We introduce SE-PLNC, a Source-Encoded PLNC scheme that is traffic pattern independent and involves coordination only among onehop neighbors, making it significantly more practical to adopt PLNC in multi-hop wireless networks. To accomplish this, SEPLNC introduces three innovations: it combines bit-level with physical-level network coding, it shifts most of the coding burden from the relay to the source of the PLNC scheme, and it leverages multi-path relaying opportunities available to a particular traffic flow. We evaluate SE-PLNC using theoretical analysis, proof-ofconcept implementation on a Universal Software Radio Peripherals (USRP) testbed, and simulations. The theoretical analysis shows the scalability of SE-PLNC and its efficiency in large adhoc networks while the testbed experiments its real-life feasibility. Large-scale simulations show that TWRC PLNC barely boosts network throughput while SE-PLNC improves it by over $30 \%$.
\end{abstract}

\section{INTRODUCTION}

Physical-Layer Network Coding (PLNC) [1] is an interference management technique that allows multiple simultaneous transmissions to a same receiver. In order to highlight its ability to increase end-to-end throughput, the community tightly affiliated this technique to the Two-Way Relay Channel (TWRC) scheme [2] depicted in Fig. 1. In this scenario, nodes $A$ and $B$ want to send a packet to each other via the relay $R$. Contrary to traditional interference-free transmissions, with PLNC, the two sources can send their packet $p_{1}$ and $p_{2}$ simultaneously to the relay node in the first time slot. By processing the superimposed received signals, node $R$ decodes a linear combination of these two packets. Typically, at bit level, it retrieves the Xor-ed packet $p_{1} \oplus p_{2}$, which it forwards to the two extremity nodes in the second time slot. Upon reception, nodes $A$ and $B$ can extract their respective messages by removing their contribution from the received coded packet. As detailed in the three configurations of Fig. 1, PLNC improves throughput by $100 \%$ and $50 \%$ compared to interference-free transmissions and the well-known packetbased Network Coding (NC) [3], respectively.

Nevertheless, these remarkable gains are inherent to the TWRC scenario and may diminish in general traffic conditions. Indeed, when bi-directional flows and TWRC elements (a relay in the middle of two opposite flows) are not frequent,

978-1-5386-4725-7

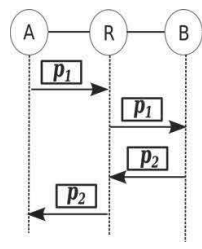

(a) IF

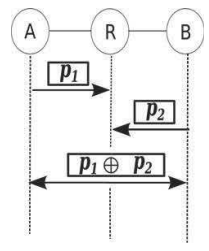

(b) $\mathrm{NC}$

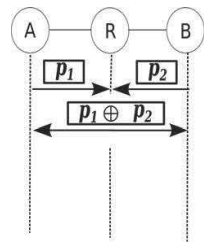

(c) PLNC
Fig. 1. TWRC scenario with a) Traditional interference-free transmissions, b) Packet-based Network Coding, c) Physical-Layer Network Coding

triggering PLNC transmissions becomes occasional, cancelling the promised benefits of such a technique. Realizing this obvious limitation, previous works have attempted to address it by introducing new PLNC configurations, such as the so called X topology [4] and Intra-Flow [2]. The former, however, is essentially an extension of TWRC and requires reliable opportunistic listening while the latter requires topologies of at least three hops and coordination between nodes two hops away.

In this paper, we consider what we believe to be a key challenge for the future of PLNC in large wireless networks: decoupling it from the canonical TWRC configuration and making it applicable to essentially any scenario in multi-hop wireless networks. To address this challenge, we introduce Source-Encoded PLNC (SE-PLNC), a scheme that relies on three innovations. First, by combining the network coding at analog and digital levels (Physical-Layer NC and packet-based NC), SE-PLNC relaxes the crossing-flows requirement from which TWRC suffers. Second, all coding/decoding operations take place among one-hop neighbors, relaxing the 3-hopminimum-path-length requirement from which the Intra-Flow scheme suffers. Third, by leveraging multi-path routing, SEPLNC is applicable to essentially any scenario in multi-hop wireless networks.

To evaluate SE-PLNC, we follow three methods. First, we conduct a geometrical analysis to highlight the scalability of our solution by quantifying its spatial reuse efficiency when associated to generic access methods. The analysis shows that SE-PLNC improves the spatial re-use efficiency by at least $20 \%$ and as much as $100 \%$ when compared to interference-free transmissions. Second, over a USRP-based testbed we develop a proof-of-concept for demonstrating the feasibility of SEPLNC on real hardware and with over-the-air transmissions. 
Experimental data show that a) our PLNC implementation outperforms a state-of-the-art implementation in terms of BitError-Rate (BER), showing a strong resilience to power level differences in the received signals, a major practical issue for PLNC and $b$ ) the performance of SE-PLNC for basic topologies matches the theoretical values, demonstrating its practical feasibility. Third, we use simulations to evaluate SEPLNC in large-scale topologies. Simulation results show that SE-PLNC outperforms both interference-free transmissions and the popular TWRC PLNC by almost $30 \%$.

The remainder of the paper is organized as follows: In Section II, we give an overview of state-of-the-art solutions aimed at integrating PLNC in large ad-hoc networks. In Section III, we introduce the Source-Encoded PLNC scheme. We exhibit the scalability of this solution in Section IV and demonstrate its feasibility in practice in Section V. Finally, simulation results are provided in Section VI. Section VII concludes this paper and describes future research perspectives.

\section{Physical-Layer Network Coding overview}

\section{A. PLNC in large multi-hop networks}

While most of the research on PLNC focuses on designing physical layer mechanisms able to support two simultaneous transmissions, few studies have been conducted to integrate PLNC in large multi-hop wireless networks. This observation can be explained by the studies [5] and [6] that prove that, whether PLNC is used or not, in a network with an infinity of flows, the per node throughput shrinks asymptotically to zero. If at first sight this pessimistic result can discourage the adoption of PLNC for large topologies, simulations performed in [4] show however that in realistic radio deployments of tens of nodes, PLNC offers considerable performance enhancement. More precisely, by allowing PLNC transmissions, limited changes to the 802.11 MAC mechanism are sufficient to increase the whole network throughput by $114 \%$ compared to interference-free transmissions.

More importantly, an extension of the existing TWRC scheme has been considered in [4] in order to address the cases when the traffic is not perfectly bi-directional. Associated to opportunistic listening, this new PLNC scheme, called "X Topology scheme" in [2], gives more opportunities to trigger PLNC transmissions when nodes do not form a TWRC topology. In the scenario of Fig. 2(a) for instance, while the TWRC scheme can not be applied, the per flow throughput is doubled with such a mechanism. Indeed, by overhearing the native packet sent by node $S 1$ (resp. node $S 2$ ) in the first slot, node $D 2$ (resp. node $D 1$ ) gathers enough information to decode the coded packet sent by the relay $R$ in the second slot. Thus, the number of necessary slots to send 2 packets is divided by two with this $\mathrm{X}$ topology scheme.

More recently, a new scheme aiming at exploiting PLNC with general traffic conditions has been studied in [7]. Called Intra-Flow PLNC, it exploits the fact that a relay knows the packet sent by the next hop since it has already received it (and previously relayed). Using this simple feature, a node can handle the coded packet through the simultaneous receptions

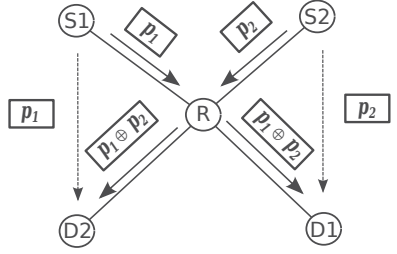

(a) X topology scheme

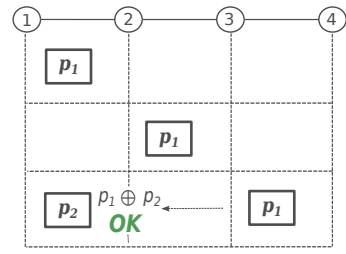

(b) Intra-Flow PLNC scheduling
Fig. 2. X topology and Intra-Flow PLNC schemes

of signals coming from its previous relay and its next relay as exemplified for node 2 in Fig. 2(b). Thus, the source of a 4-node chain topology can send 1 packet every 2 slots instead of 1 packet every 3 slots with classical interferencefree transmissions.

\section{B. Limitations of current PLNC schemes}

If the three PLNC schemes introduced in the literature (TWRC, X topology and Intra-Flow schemes) can be very beneficial in some topologies with a particular traffic pattern, they present some disadvantages that deserve to be exposed. As evoked previously, the TWRC scheme requires a strict bidirectionnal traffic to be efficient. If the "X Topology scheme" relaxes this constraint, the opportunistic listening mechanism necessary in this PLNC scheme is still an open problem in wireless networks. How can a node be sure that its neighbors have learned a packet by opportunistic receptions without adding too much overhead? Additionally, as for the TWRC PLNC, the X topology scheme is useless when flows follow the same direction.

This last point has been corrected by the Intra-Flow PLNC scenario. Assuming that each flow is composed of a batch of packets, since interfering packets belong to the same flow in the Intra-Flow PLNC scheme, whatever the traffic pattern, Intra-Flow PLNC can be used more often than the other ones as shown in [7]. Nevertheless, as in the TWRC and X topology schemes, senders are not direct neighbors what makes their coordination challenging.

Moreover, a 4-node chain is necessary to set up such a mechanism. In other words, Intra-Flow PLNC is inapplicable for 1-hop or 2-hop flows which clearly reduces its sphere of operation in real deployments.

\section{SOURCE-ENCODED PLNC}

We believe that a big reason why the significant performance gains promised by PLNC are not always realized in practice is the restrictions it imposes on the topology structure and traffic patterns. To overcome these limitations, in this section, we introduce a novel, Source-Encoded PLNC scheme (SE-PLNC) that is based on intelligently combining PhysicalLayer Network Coding (analog level) and packet-based Network Coding (digital level). First, we introduce a version of our scheme that can be employed by a single node needing to serve two traffic flows. Then, we relax the requirement for two flows and introduce an approach that works with a single 


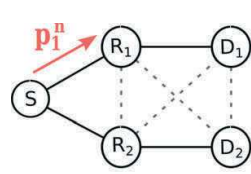

(a) Slot 1

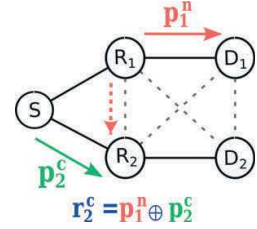

(b) Slot 2

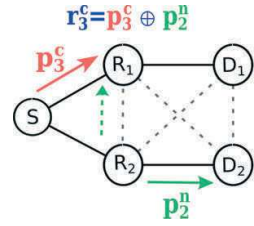

(c) Slot 3
Fig. 3. Source-Encoded PLNC - 3 slots illustration

traffic flow. For the sake of clarity, we focus in the following on small representative topologies. However, our solution can be employed in any multi-hop network where these elementary topologies can be found.

\section{A. Two-flow SE-PLNC}

Let us consider the simple scenario depicted in Fig. 3. The source, station $S$, wants to send two batches of packets to stations $D_{1}$ and $D_{2}$ via the relays $\left(R_{1}\right.$ and $\left.R_{2}\right)$, respectively. Obviously, it is impossible for $S$ to employ any of the stateof-the-art PLNC approaches to improve performance. There are no crossing flows, excluding TWRC and $\mathrm{X}$ topologies, and the paths are two-hop long, excluding Intra-Flow PLNC. Consequently, as with traditional interference-free transmissions, none of the 4 links can be active at the same time. In other words, assuming channel access is regulated by a TDMA (Time Division Multiple Access) protocol, the maximum throughput of each flow is at best 1 frame every 4 slots (with an optimal scheduling). Indeed, under the traditional interference-free scheduling, the source, $S$, transmits on the first slot a native packet, denoted $p_{1}^{n}$, to $R_{1}$ (Fig. 3(a)). In the second slot, node $R_{1}$ relays this packet to its final destination, $D_{1}$. The process is repeated between $S$ and $D_{2}$, requiring a total of 4 slots for delivering one packet to each destination.

In order to reduce this required number of slots, and thereby improve network performance, in the following we use a constructive approach to introduce the Source-Encoded PLNC (SE-PLNC). This new scheme allows station $S$ to benefit from PLNC. In particular, by enabling a PLNC transmission in the second slot, node $S$ could transmit packet $p_{2}^{n}$ with destination $D_{2}$ to $R_{2}$ while $R_{1}$ is transmitting $p_{1}^{n}$ to $D_{1}$. $R_{2}$ would receive the physically-coded packet $r_{2}^{c}$, such that $r_{2}^{c}=p_{1}^{n} \oplus p_{2}^{n}$. Unfortunately, $R_{2}$ can not retrieve its intended packet, $p_{2}^{n}$, since it does not know $p_{1}^{n}$. A potential solution could be for $R_{2}$ to opportunistically listen and receive $p_{1}^{n}$ during the first slot. However, opportunistic listening, as already argued, is not suitable for real deployments.

Our solution to this challenge is to combine bit-level Network Coding with Physical-Layer Network Coding. Specifically, as illustrated in Fig. 3(b), $S$ sends to $R_{2}$ the coded packet $p_{2}^{c}=p_{1}^{n} \oplus p_{2}^{n}$ instead of sending the native packet $p_{2}^{n}$. $R_{2}$ retrieves from the PLNC reception the packet $r_{2}^{c}=p_{1}^{n} \oplus p_{2}^{c}$. Knowing the properties of a XOR operation and since $p_{2}^{c}=$ $p_{1}^{n} \oplus p_{2}^{n}$, the received packet $r_{2}^{c}$ can be simplified as follows :

$$
r_{2}^{c}=p_{1}^{n} \oplus p_{2}^{c}=p_{1}^{n} \oplus\left(p_{1}^{n} \oplus p_{2}^{n}\right)=p_{2}^{n}
$$

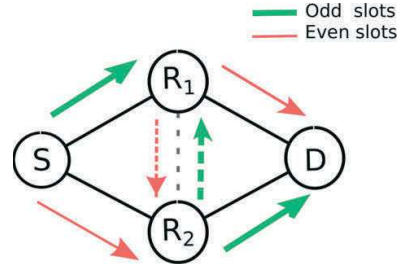

Fig. 4. Multi-path relaying combined to SE-PLNC

In other words, node $R_{2}$ does not have to execute any decoding operations since the received packet, at the bit level, is the intended native packet $p_{2}^{n}$.

$R_{2}$ can then relay $p_{2}^{n}$ to $D_{2}$ in the third slot (Fig. 3(c)). What is more, SE-PLNC allows $S$ to transmit a third packet in the very same slot. Specifically, it can send a coded packet $p_{3}^{c}=$ $p_{2}^{n} \oplus p_{3}^{n}$, ensuring that the received packet at $R_{1}$ is a native packet, $p_{3}^{n}\left(R_{1}\right.$ does not possess $\left.p_{2}^{n}\right)$. It is clear from now that $S$ can continue sending bit-level network coded packets on every slot, alternating between packets intended for $D_{1}$ and $D_{2}$. The per-flow throughput becomes 1 frame every 2 slots, doubling the performance of a traditional interference avoidance scheme.

\section{B. Single-flow SE-PLNC}

The SE-PLNC we introduced in Section III-A enables the application of PLNC in scenarios as common as that of a single node sending packets to two or more destinations. Nevertheless, at first sight, the new scheme would seem to present some of the same limitations as the TWRC and X topology schemes, namely, to be dependent on the traffic pattern. In this section, we ask the following question: can SE-PLNC be applied on the packets of a single flow so as to relax any traffic pattern requirement?

To answer this question, we combine SE-PLNC to a very simple multi-path relaying mechanism. Let us focus on the network of Fig. 4 where only one flow is established between nodes $S$ and $D$. With a classical routing protocol and traditional interference-free transmissions, the source can send at most 1 frame every 2 slots to the destination. Applying a multi-path routing protocol as it is does not change anything since with interference-free transmissions only one link can be active at a time.

However, using SE-PLNC we can leverage multi-path routing to improve performance. As in the previous scenario, the source can send one (network coded) packet on every slot alternatively to $R_{1}$ and $R_{2}$. At each relay, the signal received from the source is mixed with the signal sent by the other relay to $D$, leading to a PLNC reception. Using the combination of bit-level Network Coding at the source with the Physical-Layer Network Coding taking place naturally between the transmission of the source and the relay, the second relay retrieves its native packet. Therefore, the source is allowed to transmit a new packet on every slot, doubling its throughput, even in the case of a single flow. More importantly, assuming that multi-path relaying is feasible (quite a reasonable assumption in dense networks), this is the first scheme enabling PLNC 


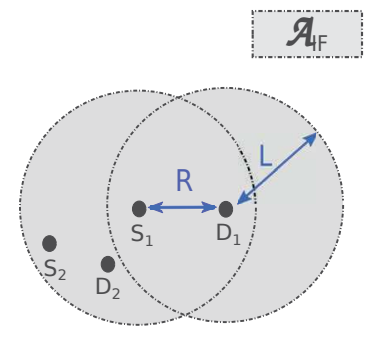

(a) Interference-free transmission

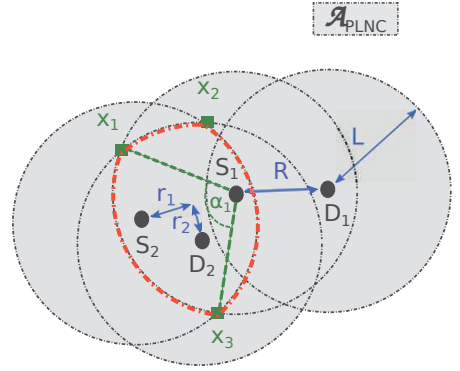

(b) SE-PLNC
Fig. 5. Reservation area illustration for a) interference-free and b) SE-PLNC transmissions

transmissions that is traffic pattern independent and works on any non-trivial path length.

\section{Scalability Study}

Our Source-Encoded PLNC, used with optimal scheduling, can increase the throughput in small topologies by $100 \%$. However, the scalability of SE-PLNC remains an open question. Does this mechanism, used with a generic access method, sustain the promised gains in wireless networks? As a part of our comprehensive answer, in the following we conduct a theoretical analysis, demonstrating that SE-PLNC presents good performance in terms of spatial reuse which justifies its integration in large multi-hop wireless networks.

\section{A. Problem formulation}

Let us generalize the two proposed SE-PLNC configurations (single-flow and two-flow) and use the SE-PLNC elementary topology shown in Fig. 5(b) composed of 4 nodes in which node $D_{2}$ performs a PLNC reception of packets sent by $S_{1}$ and $S_{2}$ (we also assume that $S_{2}$ knows the packet sent by $S_{1}$ to encode). Assuming a reliable MAC layer, meaning any scheduling which protects a DATA/ACK exchange by silencing the sender's and receiver's neighboring nodes, Fig. 5(b) shows the interference domain of the whole SE-PLNC scheme. In contrast with the traditional interference-free transmissions, $\mathcal{A}_{P L N C}$, the interference domain of the two transmissions $\left(S_{1} \rightarrow D_{1}\right.$ and $\left.S_{2} \rightarrow D_{2}\right)$, indicated by the 4 biggest circles surrounding the participating nodes, encompasses the active nodes of the concurrent communications. With SEPLNC, two nodes $\left(S_{1}\right.$ and $\left.S_{2}\right)$ can be active simultaneously in the interference area of $D_{2}$, instead of one with traditional interference-free transmissions.

As shown in Fig. 5, one can notice that the interference domain of SE-PLNC is larger than $\mathcal{A}_{I F}$, the interference domain of the interference-free transmission (Fig. 5(a)). As a result, even if the number of the simultaneous data transmissions is higher with SE-PLNC (two compared to a single transmission with traditional communications) the reservation area is increased. One has to wonder whether in large and dense deployments, gaining a single transmission at the price of silencing a higher number of nodes will not nullify the promising gains of SE-PLNC.
Therefore, we mathematically study the spatial reuse efficiency of the two communication modes to determine if SEPLNC is worth the integration in large wireless networks. Towards this, we compare the ratio between the number of exchanged packet and the interference domain for the two types of transmissions. Specifically, we compare $e_{I F}=\frac{1}{\mathcal{A}_{I F}}$ and $e_{P L N C}=\frac{2}{\mathcal{A}_{P L N C}}$ which can be seen as the number of successfully sent packets per square unit in the two cases.

\section{B. Spatial Reuse Computation}

To compute the spatial reuse, we first need to obtain $\mathcal{A}_{I F}$ and $\mathcal{A}_{P L N C}$, the interference domains for interference-free and PLNC transmissions, respectively. Let us denote with $R$ the communication range and $L$ the interference range; by definition, $L \geq R$. Classically, the circle centered at the source with radius $R$ (resp. $L$ ) constitutes the communication domain (resp. interference domain). While it is well established that the radio transmission footprint does not constitute a perfect circle, such an assumption can be made in our case since we are comparing the two considered approaches under similar conditions.

In order to compute $\mathcal{A}_{I F}$ and $\mathcal{A}_{P L N C}$, we reduce the number of variables by fixing in the two cases the distance between $S_{1}$ and $D_{1}$ to $R$ representing the maximal communication distance. One can easily see that $\mathcal{A}_{I F}$ can be written as :

$$
\begin{aligned}
\mathcal{A}_{I F} & =2 \Pi L^{2}-\left[2 L^{2} \arccos \left(\frac{R}{2 L}\right)-R \sqrt{L^{2}-\frac{R^{2}}{2}}\right] \\
& =2 \Pi L^{2}-f_{S_{1}, D_{1}}(L)
\end{aligned}
$$

with $f_{X, Y}(Z)$ the area of the intersection lens of the two circles of centers $X$ and $Y$ and radius $Z$. Here $f_{S_{1}, D_{1}}(L)$ denotes the lens of circles centered at $S_{1}$ and $D_{1}$ with radius $L$.

$\mathcal{A}_{P L N C}$ value depends also on the positions of nodes $S_{2}$ and $D_{2}$. We denote with $r_{1}$ half of the distance between $S_{1}$ and $S_{2}$. As $S_{2}$ knows the packet sent by $S_{1}$ in our SE-PLNC, we assume that $S_{1}$ and $S_{2}$ are direct neighbors. Since PLNC has better performance when the received powers of the two signals are similar (see Section V), we consider that node $D_{2}$ is at an equal distance from $S_{1}$ and $S_{2}$. More formally, $D_{2}$ is on the perpendicular bisector of the $S_{1} S_{2}$ line segment. We also denote with $r_{2}$ the distance between $D_{2}$ and this line segment as shown in Fig. 5(b). With these, $\mathcal{A}_{P L N C}$ can be upper bounded by the following expression:

$$
\begin{gathered}
\mathcal{A}_{P L N C}< \\
4 \Pi L^{2}-\left[f_{S_{1}, D_{1}}(L)+f_{S_{1}, S_{2}}(L)+f_{S_{1}, D_{2}}(L)+f_{S_{2}, D_{2}}(L)\right] \\
+g_{S_{1}, S_{2}, D_{2}}(L)
\end{gathered}
$$

$g_{S_{1}, S_{2}, D_{2}}(L)$ is the intersection area of 3 circles of radius $\mathrm{L}$ and centers $S_{1}, S_{2}$ and $D_{2}$ (area delimited by red contours in Fig. 5(b)). It can be computed as follows :

$$
g_{S_{1}, S_{2}, D_{2}}(L)=\nabla_{x_{1}, x_{2}, x_{3}}+\sum_{i=1}^{3} \frac{L^{2}}{2}\left(\alpha_{i}-\sin \alpha_{i}\right)
$$

$x_{1}, x_{2}$ and $x_{3}$ are the intersection points of the three studied circles and $\nabla_{x_{1}, x_{2}, x_{3}}$ is the triangle $x_{1} x_{2} x_{3}$ area. In the interest 


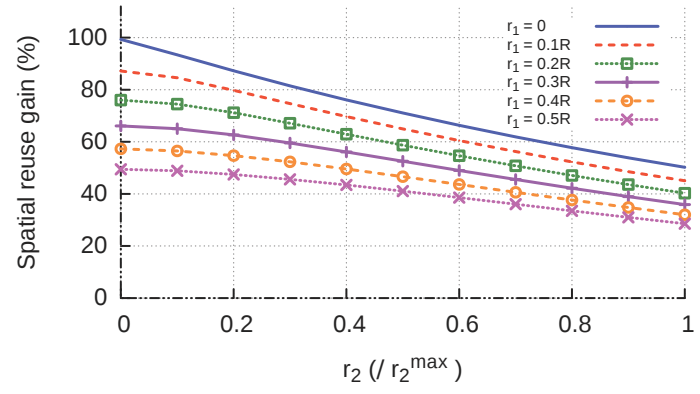

Fig. 6. Gains in terms of spatial reuse of SE-PLNC compared to interferencefree transmissions

of clarity, we do not provide the full mathematical expressions with the exact coordinates of these three points which can be expressed as function of $r_{1}, r_{2}, L$ and $R$. Similarly, the $\alpha_{1}$ (resp. $\alpha_{2}, \alpha_{3}$ ) expression, which represents the angle $\widehat{x_{1} S_{1} x_{3}}$ (resp. $\widehat{x_{1} D_{2} x_{2}}, \widehat{x_{2} S_{2} x_{3}}$ ), is not detailed neither. However, some of these parameters are highlighted in Fig. 5(b) for easier understanding.

Given $\mathcal{A}_{I F}$ and the $\mathcal{A}_{P L N C}$ upper bound expression, we can now evaluate the gains in terms of spatial reuse of SE-PLNC by evaluating the $\frac{e_{P L N C}}{e_{I F}}$ ratio for different values of $r_{1}$ and $r_{2}$. In particular, we vary $r_{1}$ from 0 to $\frac{R}{2}$ and $r_{2}$ from 0 to $r_{2}^{\max }=$ $\sqrt{R^{2}-r_{1}^{2}}$ in order to guarantee the neighboring constraints. In practice, $r_{2}^{\max }$ condition ensures that $D_{2}$ remains in the communication range of $S_{1}$ and $S_{2}$. Then, in order to protect the weakest transmissions (the longest in terms of distance), we classically choose a reservation range $25 \%$ larger than the data transmission range $(L=1.25 \times R)$.

Fig. 6 shows the spatial reuse gains for different positions of $S_{2}$ and $D_{2}$ inside the $S_{1} \rightarrow D_{1}$ interference domain. For every $S_{2}$ position (i.e. $r_{1}$ value) we move $D_{2}$ over the perpendicular bisector (i.e consider different normalized values of $r_{2}$ ) until the neighboring constraint is no longer satisfied. Results depicted in Fig. 6 show that regardless of the positions of nodes $S_{2}$ and $D_{2}$, the number of packets sent per reserved unit square area is at least $20 \%$ higher with SE-PLNC when compared to traditional transmissions. As expected, the closer $S_{2}$ and $D_{2}$ to $S_{1}$ are, the better is the spatial reuse. Indeed, the reservation areas of the three nodes merge, reducing the interference domain of the whole system, allowing more communication opportunities to neighboring nodes. In summary, the results confirm that the proposed SEPLNC offers better spatial reuse regardless of the position of the two communicating node couples. Interestingly for a scalability study, the denser the topology, the higher the expected gains.

\section{EXPERIMENTAL EVALUATION}

We detail in this part our elaborated proof-of-concept based on a USRP testbed. We further highlight in a realistic experimental environment the feasibility of SE-PLNC in both, the two-flow and single-flow configuration, as introduced in Section III.

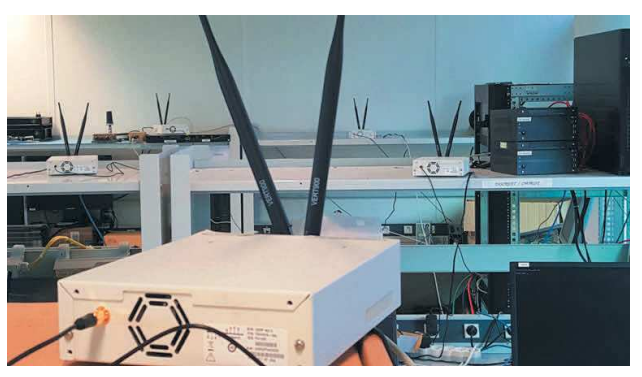

Fig. 7. USRP testbed used for the two-flow SE-PLNC evaluation. All transmissions are over the air.

\section{A. USRP Testbed}

To evaluate SE-PLNC, we use the testbed depicted in Fig. 7. It consists of 5 Ettus Research Universal Software Radio Peripherals (USRPs) running the GNU Radio framework [8]. Each radio is a USRP N210 equipped with a SBX daughterboard and GPS antennas. Communications take place over the $1.8 \mathrm{GHz}$ frequency band with a sampling rate of $400 \mathrm{kB} / \mathrm{s}$. A slotted TDMA framework is built thanks to the GPS synchronization whereby transmissions are performed in dedicated time slots as we detail hereafter.

\section{B. PLNC Implementation}

We present our physical layer design and implementation using the topology of Fig. 8 as an illustration. In this scenario, node $C$ tries to decode the XOR-ed packet of the two native packets $p_{A}$ and $p_{B}$ sent by nodes $A$ and $B$, respectively. To do so, as in [9] and [10], we use an Orthogonal FrequencyDivision Multiplexing (OFDM) coding structure as it can reduce the impact of signal misalignment. In each OFDM symbol, 64 subcarriers are used: 40 data subcarriers for the effective data transfers i.e. sBPSK (Binary Phase-Shift Keying) modulated symbols, a DC (Direct Current) carrier with no information on it, 11 guard carriers and 12 pilot carriers used to track the channel evolution during the transmission. A cyclic prefix (CP) of 16 samples is added to absorb the symbols misalignment. Consequently, the symbol timing offset in the time domain shifts to a phase rotation in the frequency domain, which can be corrected by channel estimation and equalization, as described below.

As also highlighted in Fig. 8, the simultaneous payload transmission of nodes $A$ and $B$ is preceded by 2 headers consisting of 3 OFDM symbols each (2 pilot symbols and 1 metadata symbol) sent alternatively (orthogonalization in time). They are used by node $C$ to get crucial information required for the data part decoding [11].

Interference-free header part: The first pilot symbol is used to estimate the Channel Frequency Offsets between node $C$ and the two senders $\left(C F O_{A}\right.$ with node $A$ and $C F O_{B}$ with node $B$ ). Indeed, even if the external received GPS signal ensures a good frequency synchronization between all the three nodes, some inaccuracies from hardware imperfection are persistent and need to be corrected for better performance. For this reason, we implement the popular Schmild and Cox 


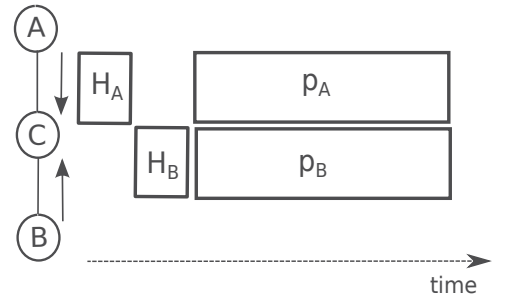

Fig. 8. Physical layer design

TABLE I

Node C Decoding Decision Table

\begin{tabular}{|c|c|}
\hline$\left(\epsilon_{A}, \epsilon_{B}\right)=\underset{\substack{\epsilon_{A} \in(-1,+1) \\
\epsilon_{B} \in(-1,+1)}}{\operatorname{argmin}}\left|Y_{C}^{m, k}-\left(\epsilon_{A} H_{A}^{m, k}-\epsilon_{B} H_{B}^{m, k}\right)\right|^{2}$ & $\hat{s}^{m, k}$ \\
\hline$(-1,-1)$ & 0 \\
\hline$(-1,+1)$ & 1 \\
\hline$(+1,-1)$ & 0 \\
\hline$(+1,+1)$ & \\
\hline
\end{tabular}

algorithm [12]. Briefly, this algorithm, based on the symmetry of the first training symbol, compares the received phase of the two halves of this symbol in the time domain to estimate the frequency offset.

Contrary to the CFO estimation, the channel estimation is performed in the frequency domain, after the Fast Fourier Transform (FFT) operation and the $\mathrm{CP}$ removal, thanks to the second pilot symbol. This OFDM symbol is a pseudorandom sequence such that each value transmitted on the data subcarriers belongs to $(-1,1)$, and 0 elsewhere (non-data subcarriers). Denoting with $X_{A}^{m_{0}, k}$ the symbol transmitted by node $A$ and $Y_{A}^{m_{0}, k}$ the same symbol as received at node $C$ on the $k$-th subcarrier, node $C$ estimates the channel coefficient $\hat{H}_{A}^{m_{0}, k}$ by the simple Zero-Forcing algorithm as follows:

$$
\hat{H}_{A}^{m_{0}, k}=\frac{Y_{A}^{m_{0}, k}}{X_{A}^{m_{0}, k}}
$$

Note that, the channel coefficients estimated during the header reception may change during the data payload transmission. Therefore, it is important to track the channel variation during the interfering data part. This is the role of pilot subcarriers contained in each data OFDM symbol. By allocating in an orthogonal way 6 of the 12 pilot subcarriers to each sender, we ensure that node $C$ receives non-interfering symbols on each pilot subcarrier. With this, even during the data transmission part, it can estimate the channel variation at the $m$-th OFDM symbol for each pilot subcarrier, $p$, as follows :

$$
\Delta \hat{H}_{A}^{m, p}=\frac{Y_{A}^{m, p}}{\hat{H}_{A}^{m_{0}, p} X_{A}^{m, p}}
$$

Then, node $C$ can estimate the channel coefficients of the $m$-th OFDM symbol for each data subcarrier $k$ as follows:

$$
\hat{H}_{A}^{m, k}=\Delta \hat{H}_{A}^{m, k} \hat{H}_{A}^{m_{0}, k}
$$

where $\Delta \hat{H}_{A}^{m, k}$ is obtained by a linear interpolation with the two closest pilot subcarriers $p_{1}$ and $p_{2}$, as follows :

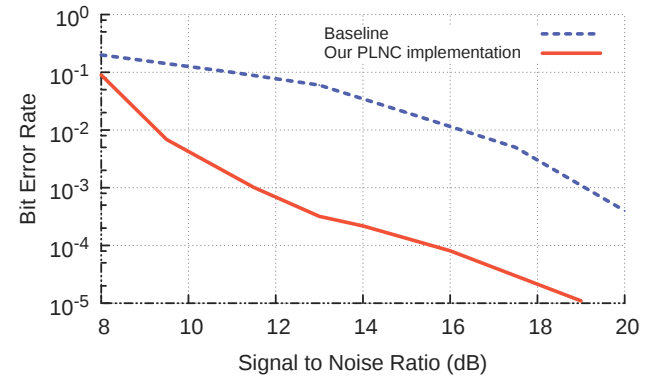

(a) Equal received power

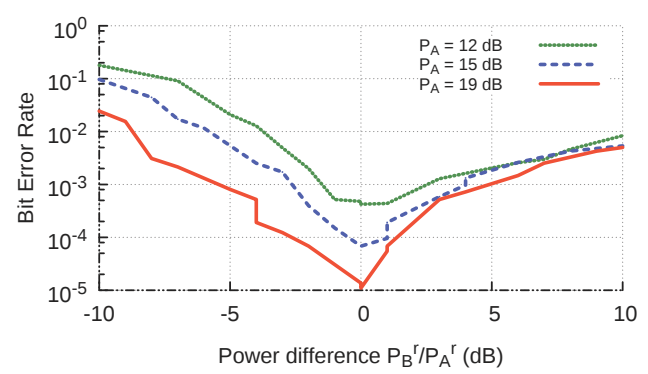

(b) Different received power

Fig. 9. Our PLNC implementation performance - a) Equal received power and b) Different received power

$$
\Delta \hat{H}_{A}^{m, k}=\Delta \hat{H}_{A}^{m, p_{1}}+\left(k-p_{1}\right) \frac{\Delta \hat{H}_{A}^{m, p_{2}}-\Delta \hat{H}_{A}^{m, p_{1}}}{p_{2}-p_{1}}
$$

To have a more precise channel estimation, while keeping the pilot subcarriers orthogonalization, we vary the position of pilot subcarriers for two successive OFDM symbols.

Finally, the third interference-free symbol in the header part contains metadata information, such as the packet identifier and the length of the following payload.

Simultaneous payload reception: During the payload reception, node $C$ performs the signal equalization in order to retrieve the coded packet $p_{A} \oplus p_{B}$. The first step is correcting the frequency offsets measured thanks to the first pilot symbol in the header part. To do so, before the FFT operation, as suggested in [13], each received sample is rotated by the phase induced by the mean of the frequency offset. In particular, denoting by $y_{n}$ the $n$-th received sample in the time domain, the sample $\tilde{y}_{n}$ provided to the FFT is given by the following expression:

$$
\tilde{y}_{n}=y_{n} \times e^{-2 j n \Pi \frac{C F O_{A}+C F O_{B}}{2} \frac{T}{N}}
$$

where $T$ is the duration of an OFDM symbol and $N$ the number of samples in an OFDM symbol.

Once the frequency offset corrected, the FFT and the CP removal applied, each data symbol decision is performed in the frequency domain following the minimum distance criteria. Using the channel coefficients estimation obtained via the second pilot symbol of the headers and the pilot subcarriers, node $C$ estimates the symbol $\hat{s}^{m, k}$ from the mixed symbol $Y_{C}^{m, k}$ received in the $m$-th OFDM symbol on subcarrier $k$, following the rules depicted in Table I. 


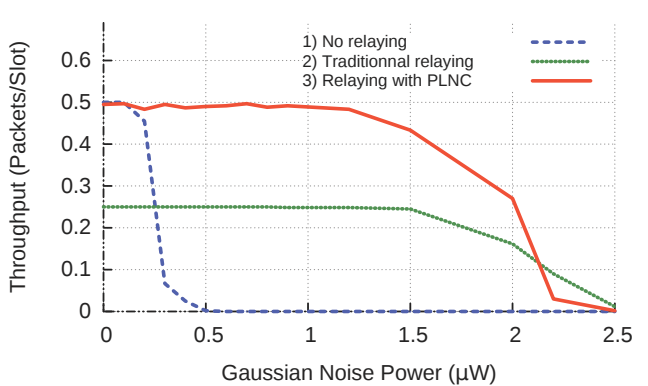

Fig. 10. Implementation results of the two-flow SE-PLNC

\section{Evaluation of the PLNC implementation}

Fig. 9(a) compares with over the air transmissions the Bit Error Rate (BER) of our implementation to a baseline solution introduced in [13]. In the topology of Fig. 8, we position $C$ in a way to receive the same level of power $\left(P_{A}^{r}\right.$ and $\left.P_{B}^{r}\right)$ from the two senders. The BER values are obtained by comparing the packet decoded by node $C$ to the $p_{A} \oplus p_{B}$ packet. The data shows that our PLNC implementation outperforms the baseline, thanks to the tighter MAC-level synchronization we have obtained by leveraging the GPS signal.

Even if the MAC-level synchronization is addressed, PLNC is still considered vulnerable to the difference in power levels of the two received signals. To quantify this impact on our implementation, we fix at $C$ the received power from $A$ transmission $P_{A}^{r}(12 \mathrm{~dB}, 15 \mathrm{~dB}, 19 \mathrm{~dB})$ and vary $P_{B}^{r}$ by adapting the transmitted power of node $B$ (nodes $A$ and $B$ are placed at equal distance from $C$ ). Whereas the existing implementations are shown to be inefficient when the difference in received signal powers is more than $1 \mathrm{~dB}$ [9], Fig. 9(b) shows that our implementation can tolerate much bigger values. For $P_{A}^{r}=19 d B$ for instance, the BER is less than $10^{-3}$, sufficient for transmitting packets of reasonable sizes, even when the difference in received power levels is $5 \mathrm{~dB}$.

\section{Evaluation of the two-flow SE-PLNC}

In this first experiment, we reproduce the scenario of Fig. 3 in which the source node $\mathrm{S}$ wants to send two batches of 1280 bit-sized packets to 2 destinations (nodes $D_{1}$ and $D_{2}$ ).

We compare 3 different scenarios: 1) Direct transmissions from $S$ to the two destinations, 2) Relaying through $R_{1}$ and $R_{2}$ using only traditional interference-free transmissions, 3) Our fully implemented two-flow SE-PLNC introduced in Section III-A. In the 3 cases, we use optimal scheduling. In the first scenario, the maximum source rate is equal to 0.5 frame per slot per flow since the source sends packets to $D_{1}$ and $D_{2}$ alternatively. In the second scenario, the 4 necessary transmissions cannot coexist and the source can send 1 packet every 4 slots for each flow, at best. With SE-PLNC, the source node transmits one packet to $R_{1}$ (resp. $R_{2}$ ) and $R_{2}$ (resp. $R_{1}$ ) relays the last received packet to the destination $D_{2}$ (resp. $D_{1}$ ) in the odd slots (resp. even slots) leading to a source rate of 0.5 frame per slot per flow. In all scenarios, we do not add any correction codes, which means that every erroneous bit leads to a cyclic redundancy check (CRC) failure of the packet.

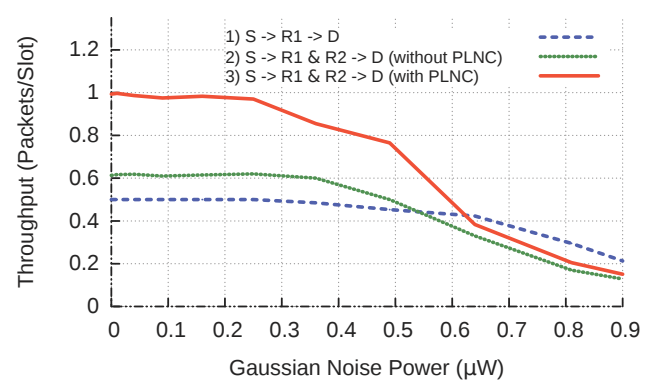

Fig. 11. Performance of the SE-PLNC-based multi-path relaying strategy

To compare the three strategies, we evaluate the number of error-free packets received by $D_{1}$ after 1000 slots of operation (Fig. 10). The $x$-axis shows the power of an artificial Gaussian noise we add at the two destinations to increase signal distortion. This was done to emulate the effect of increasing the distance between the source and $D_{1}, D_{2}$, which we could not physically realize due to lack of physical space in our laboratory. Note that all transmissions are over the air. We do not show the results for node $D_{2}$ since they are similar to those for $D_{1}$.

Fig. 10 shows that with little added noise at the receivers, direct transmissions between $S$ and $D_{1}$ are possible, allowing the latter to receive 1 packet every 2 slots (maximum throughput). As the noise level increases, direct transmissions become inefficient, making relaying through $R_{1}$ and $R_{2}$ necessary. In this case, SE-PLNC achieves a $100 \%$ improvement when compared to interference-free transmissions. Interference-free transmissions cannot achieve throughput higher than 0.25 frame per slot compared to 0.5 frame per slot with our PLNC scheme.

Because of the additional 3-OFDM-symbol header and the multiplication by 2 of the pilot subcarriers number, the slot duration for sending a PLNC packet is $21 \%$ larger than the slot duration of an interference-free transmission. This constitutes the overhead of our PLNC implementation. Note that this overhead can still be optimized by increasing the packet size or reducing the number of pilot carriers; our evaluation was mainly aiming at feasibility studies. Nevertheless, even in this configuration, the gains of our SE-PLNC remain highly significant.

\section{E. Evaluation of the single-flow SE-PLNC}

In the second experiment, we evaluate the performance of multi-path relaying with PLNC transmissions. For this, we set up the exact scenario of Fig. 4 where the source node $S$ has packets to transmit to node $D$. We compare the received throughput when 1) packets are relayed through $R_{1}$ only (source rate $=0.5$ packet per slot), 2) packets are routed alternatively through $R_{1}$ and $R_{2}$ with traditional signal equalization at relays (source rate $=1$ packet per slot) and 3) SE-PLNC (source rate $=1$ packet per slot). Fig. 11 shows that the multi-path SE-PLNC clearly outperforms the other relaying strategies. Indeed, limited by its source rate, the classical one-path approach cannot achieve higher throughput 


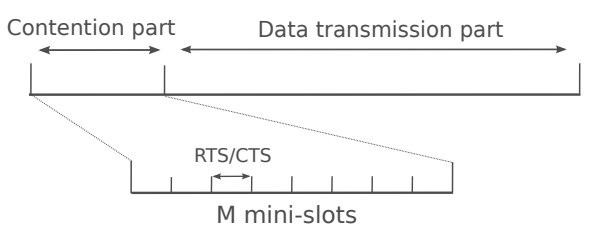

Fig. 12. TDMA slot design: Contention and data transmission parts

than 0.5 frame per slot. The multi-path approach, with traditional interference-free signal processing at relays, has better performance since the source transmits packets in every slot. However, most packets sent from the source to a relay are lost because of the interfering packets transmitted simultaneously by the other relay. This issue is solved with the multi-path SEPLNC, resulting in a $100 \%$ improvement when compared to the traditional relaying approach and $65 \%$ when compared to multi-path relaying without PLNC. Even accounting for the overhead induced by our PLNC implementation, the performance of our system remains largely significant.

\section{Performance in A SLOtTed 802.11-BASED NETWORK}

The geometrical study of Section IV indicates the reasons for which the developed SE-PLNC should be efficient in large multi-hop networks with realistic scheduling mechanisms. Of course, such an affirmation deserves to be validated by simulation results of large scale deployments.

\section{A. Scheduling mechanism description}

Evaluating the performance of the developed SE-PLNC in large environment requires combining this technique to an existing TDMA access mechanism. Indeed, the implementation described above is based on a TDMA MAC layer, and more generally PLNC concept has better performance in such synchronous systems. Then, as in [14], we choose to adopt a slotted version of the 802.11 DCF protocol.

The considered TDMA slot design is represented in Fig. 12. Each slot is composed of two parts: a contention part for allocating the channel in a distributed manner and a data transmission period. The contention period is divided into $\mathrm{M}$ mini-slots (overhead) designed such as to allow the traditional 802.11 RTS/CTS exchange to take place. Nodes compete in this channel reservation period in order to win the access for the payload transmission which becomes naturally collisionfree. For this, in each mini-slot, a node which has a packet to transmit and which does not overhear any signal in the previous mini-slots, sends an RTS packet with a probability $\alpha$. The destination replies in the second part of the same mini-slot with a CTS packet if and only if it did not receive anything before. Then, a source node participates to the data transmission (in the contention-free period) once the RTS/CTS is done correctly. The choice of the $\alpha$ parameter is crucial in order to limit collisions during the competition part while keeping a good bandwidth utilization. A lot of algorithms based on nodes queue sizes have been developed in the last years in order to determine the optimal $\alpha$ value [14] [15].
However, this is beyond the scope of this paper and we will use the same constant value for all the nodes of the network.

Quite logically, this scheduling mechanism is specially designed for interference-free transmissions. However, a slight modification of the algorithm is sufficient to adapt it to SEPLNC. In particular, whereas in the original version a node stops to compete when it hears a reservation request, our scheduling mechanism allows nodes that receive a RTS packet and detect a PLNC opportunity to participate in the channel access race. These nodes send their RTS in the immediate following mini-slot after the detection. To illustrate such scenario, let us go back to the second slot of the two-flows scenario (Fig. 3(b)) in which node $R_{1}$ wants to send $p_{1}$ to $D_{1}$ and $S$ has a packet $p_{2}$ to $R_{2}$. Let us consider that $R_{1}$ transmits a RTS packet to $D$ in the first mini-slot. With the original version of slotted 802.11 protocol, node $S$ differs its access when it detects this RTS packet. With our adaptation, $S$ spots a PLNC opportunity by overhearing that $R_{1}$ plans to transmit packet $p_{1}$ (we assume that the packet identifier is contained into the RTS packet). Indeed, it already knows this packet and can encode it with the packet $p_{2}$ intended to $R_{2}$. Consequently, $S$ transmits a RTS packet to $R_{2}$ in the immediate next mini-slot (minislot 2) which in turn replies with a CTS since it knows that decoding the simultaneous PLNC reception of packets from $R_{1}$ and $S$ will be possible. Following this reservation, $R_{1}$ and $S$ can transmit simultaneously in the data transmission part using the OFDM structure depicted in Fig. 8.

\section{B. Performance in a Large Topology}

We evaluate the gains of SE-PLNC using the modified version of the slotted 802.11 mechanism described above. We compare our results to the original interference-free solution on the 50-node network shown in Fig. 13. To ensure fairness, the two scheduling mechanisms have the same overhead, namely 8 mini-slots of the competition part and the same $\alpha$ value $(\alpha=0.1)$. Furthermore, we also compare SE-PLNC performance to the TWRC scheme implemented with a similar access policy.

Methodology: We vary the number of traffic flows in the network from 5 to 30 and assign 2000 packets uniformly. For a given number of flows, we select the source, destination pairs uniformly at random among all the network nodes. The selection of source-destination pairs for a given number of flows is repeated 30 times in order to show the average performance across the 30 simulations. As performance metric, we use the number of packets received by all the destinations after 3000 slots of simulation. Since we believe that it can be greatly enhanced with an optimized physical layer design, we do not take into account the overhead introduced for supporting PLNC transmissions.

Then, it should be noted that the source and destination nodes of a SE-PLNC exchange are not necessary source or destination of an established flow. In particular, for flows longer than 2 hops, relays can also trigger SE-PLNC transmissions with other relays. The only constraint is that the first node involved in the SE-PLNC scheme has already received 


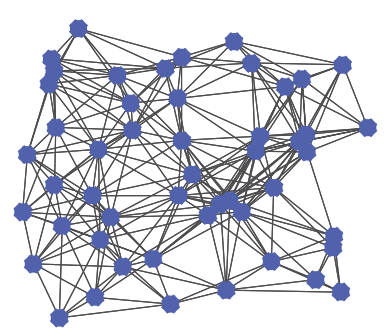

Fig. 13. 50-node topology

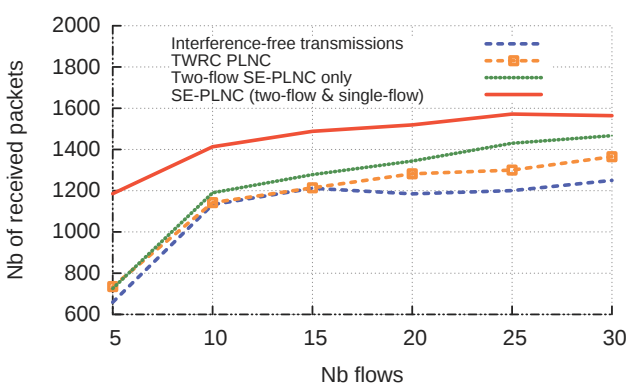

Fig. 14. Number of packets received after 3000 slots of simulation.

the packet sent by the second node. This is required in order to allow the packet-based network coding at source proposed with our solution.

Finally, the SE-PLNC scheme is evaluated with the singleflow mode enabled and disabled.

Results: Fig. 14 points to two conclusions. First, with a few number of established flows, the only PLNC-based scheme to outperform interference-free scheduling is the single-flow SE-PLNC we introduced in Sec. III-B. With few flows, the probability that two of them share the same node is low, explaining why the two-flow SE-PLNC scheme brings very little benefit. However, empowering SE-PLNC with multi-path relaying (the single-flow approach), delivers over $40 \%$ more data packets, even with only 5 traffic flows. Indeed, since SE-PLNC is applied between packets of the same flow, its performance becomes traffic independent.

Second, SE-PLNC clearly outperforms TWRC. Indeed, while using a similar MAC mechanism, TWRC is barely offering any gains over the traditional interference-free transmissions. The result looks surprising at first but, looking more carefully into the simulation data, we found that TWRC is hardly triggering any PLNC transmissions. As corroborated in Fig. 15, the total number of successful transmissions during the simulation experiment is essentially the same for TWRC and interference-free strategies. Intuitively, this can be explained by the fact that TWRC requires flows to share 3 consecutive nodes, a difficult requirement to meet even in a large network with a big number of traffic flows. Finally, with a large number of flows, although combining PLNC and multi-path relaying is not as crucial as for the low loaded regime, SE-PLNC gains remain remarkable (more than $30 \%$ for 25 established flows).

\section{ViI. CONCLUSiOn AND Future WORK}

We have proposed the SE-PLNC scheme that overcomes PLNC traffic constraints by combining bit-level Network

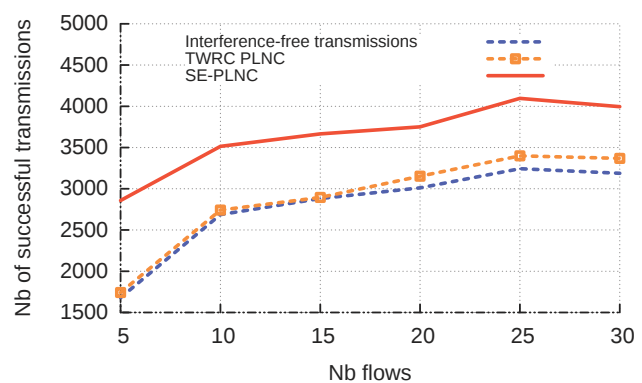

Fig. 15. Number of successful transmissions during the simulation experiment.

Coding with Physical-Layer Network Coding. Our large and gradual evaluation methodology included geometric analysis for scalability investigation, experimental testbed validations for credibility as well as simulations with adapted access technique for large scale performance. All our evaluation techniques show that SE-PLNC brings significant gains when compared to interference-free transmissions and TWRC PLNC. These gains are at least $30 \%$ in the most generic simulation scenario and reach $100 \%$ in many representative cases.

In the future, we intend to work on an asynchronous SE-PLNC mode so as to relax the TDMA MAC constraint imposed by our current design.

\section{REFERENCES}

[1] S. Zhang, S. Liew, and P. Lam, "Hot topic: physical-layer network coding," in Proc. ACM MobiCom Conference, 2006.

[2] S. Katti, S. Gollakota, and D. Katabi, "Embracing wireless interference: Analog network coding," in Proc. ACM SIGCOMM Conference, 2007.

[3] S. Katti, H. Rahul, W. Hu, D. Katabi, M. Medard, and J. CrowCroft, "Xor's in the air : Practical wireless network coding," in Proc. ACM SIGCOMM Conference, 2006.

[4] W. Mao, X. Wang, A. Tang, and H. Qian, "Anc-era: Random access for analog network coding in wireless networks," IEEE Transactions on Mobile Computing, Vol.15, No.1, pp.45 - 59, 2016.

[5] K. Lu, S. Fu, Y. Qian, and H. Shen, "On capacity of random wireless networks with physical-layer network coding," IEEE Journal on Selected Areas in Communications (JSAC), Vol.27, No.5, 2009.

[6] T. Zhang, K. Lu, A. Jafari, and S. Fu, "On the capacity bounds of large-scale wireless network with physical-layer network coding under the generalized physical model," in Proc. IEEE ICC Conference, 2010.

[7] R. Naves, H. Khalife, G. Jakllari, V. Conan, and A.-L. Beylot, "A framework for evaluating physical-layer network coding gains in multihop wireless network," in Proc. IEEE INFOCOM Conference, 2017.

[8] "Gnuradio overview available at http://www.gnuradio.org/."

[9] L. Lu, L. You, Q. Yang, T. Wang, M. Zhang, S. Zhang, and S. Liew, "Real-time implementation of physical-layer network coding," in Proc. ACM SIGCOMM SRIF Workshop, 2013.

[10] Y. Tan, S. Liew, and T.Huang, "Mobile lattice-coded physical-layer network coding with practical channel alignment," IEEE Transactions on Mobile Computing, 2016.

[11] T. Hynek, D. Halls, and J. Sykora, "Hardware implementation of distributed learning algorithm for mapping selection for wireless physical layer network coding," in Proc. IEEE ICC Workshop (ICCW) 2015 , 2015.

[12] T. M. Schmild and D. C. Cox, "Robust frequency and timing synchonization for ofdm," IEEE Transactions on Communications, Vol. 45, No. 12, 1997.

[13] L. Lu, "Asynchronous physical-layer network coding," 2012.

[14] J. Ni, B. Tan, and R. Srikant, "Q-csma: Queue-length-based csma/ca algorithms for achieving maximum throughput and low delay in wireless networks," IEEE Transactions on Networking, Vol. 20, No. 3, 2012.

[15] C. Joo and M. Shin, "Queue-affectance-based scheduling in multi-hop wireless networks under sinr interference constraints," in Proc. IEEE INFOCOM Conference, 2016. 